\title{
Effects of bile acids on biliary epithelial cell proliferation and portal fibroblast activation using rat liver slices
}

\author{
Haude Clouzeau-Girard ${ }^{1,2, *}$, Christelle Guyot ${ }^{1,2, *}$, Chantal Combe ${ }^{1,2}$, \\ Valérie Moronvalle-Halley ${ }^{3}$, Chantal Housset ${ }^{4}$, Thierry Lamireau ${ }^{1,5}$, \\ Jean Rosenbaum ${ }^{1,2}$ and Alexis Desmoulière ${ }^{1,2}$ \\ ${ }^{1}$ INSERM E0362, Université Victor Segalen Bordeaux 2, Bordeaux, France; ${ }^{2}$ IFR 66, Bordeaux, France; \\ ${ }^{3}$ Sanofi-Aventis, Alfortville, France; ${ }^{4}$ INSERM U402, Université Pierre et Marie Curie Paris 6, Paris, France \\ and ${ }^{5} \mathrm{CHU}$ Bordeaux, Hôpital des Enfants, Unité de Gastroentérologie Pédiatrique, Bordeaux, France
}

\begin{abstract}
During cholestasis, bile acids accumulate in the liver, and induce cellular alterations. Cholestasis is a major cause of liver fibrosis. We have used precision-cut liver slices (PCLS) in culture to investigate the effects of bile acids on hepatic cells. Rat PCLS were placed on an insert in a vial containing culture medium, and gently agitated on a roller platform. PCLS were treated with $100 \mu \mathrm{M}$ taurolithocholate (TLC), taurodeoxycholate (TDC) or taurocholate (TC) for 24 or $48 \mathrm{~h}$. PCLS viability was measured, and immunohistochemistry was performed with antibodies against active caspase 3, platelet-derived growth factor (PDGF) receptor- $\beta$ and ED-A fibronectin. TDC and TLC, two hydrophobic bile acids, induced hepatocyte necrosis and apoptosis, whereas TC, an hydrophilic bile acid, improved slice viability as compared with controls. Both TDC and TC induced biliary epithelial cell proliferation, together with portal fibroblast proliferation and activation, as shown by PDGF receptor- $\beta$ and ED-A fibronectin expression. TLC induced biliary epithelial cell apoptosis. Our results indicate that individual bile acids induce cell type-specific effects in a complex liver microenvironment. The fact that PCLS support biliary epithelial cell and portal fibroblast proliferation will make this model very useful for the study of the mechanisms involved in portal fibrosis.
\end{abstract}

Laboratory Investigation (2006) 86, 275-285. doi:10.1038/labinvest.3700386; published online 9 January 2006

Keywords: bile acid; biliary epithelial cell; cholestasis; liver fibrosis; liver slice; portal fibroblast

Bile acids are the most abundant biliary component and play a major role in the maintenance of bile flow. They are natural detergents and allow lipids to be digested in the intestinal lumen. During chronic cholestasis, bile acids may accumulate, particularly in hepatocytes, leading to histological abnormalities. ${ }^{1}$ This occurs especially in childhood diseases such as biliary atresia, cystic fibrosis and paucity of interlobular bile ducts, ${ }^{2}$ and also in the liver of adult patients suffering from chronic liver diseases. ${ }^{3}$ Accumulation of toxic bile acids results in hepatocellular damage, ultimately leading to liver fibrosis and cirrhosis. Bile acid cytotoxicity has been demonstrated on hepatocytes in vitro ${ }^{4,5}$ and in vivo

Correspondence: Dr A Desmoulière, PhD, GREF, INSERM E0362, Université Victor Segalen Bordeaux 2, 146 rue Léo-Saignat, 33076 Bordeaux cedex, France.

E-mail: Alexis.Desmouliere@gref.u-bordeaux2.fr

*These two authors equally contributed to this work.

Received 7 November 2005; revised 30 November 2005; accepted

2 December 2005; published online 9 January 2006 in rat models, ${ }^{6,7}$ and they are known to induce hepatocyte apoptosis. ${ }^{8}$ There is generally a correlation between bile acid hydrophobicity and cytotoxicity: the hydrophobic lithocholic and deoxycholic acids exert the most toxic effects, whereas the hydrophilic ursodeoxycholic and cholic acids have little toxicity.

During fibrogenesis, the excess extracellular matrix is synthesized by myofibroblastic cells. They derive from precursor cells, the best studied being hepatic stellate cells (for review, see ${ }^{9}$ ). However, in biliary fibrosis, experimental models such as bile duct ligation have identified portal fibroblasts rather than hepatic stellate cells as the major mesenchymal cells involved in the early stages of fibrosis development. ${ }^{10-12}$ Bile duct proliferation is a hallmark of human cholestatic disorders ${ }^{13}$ and is also observed in the bile duct ligation model..$^{14,15}$ It is accompanied by biliary epithelial cell alterations. The relative contribution of enhanced hydrostatic pressure in bile ducts ${ }^{16}$ and of increased concentrations of cytotoxic bile acids ${ }^{17}$ in the mechanisms involved 
in biliary epithelial cell damage and bile duct proliferation is unknown. Bile duct obstruction is also accompanied by a rapid activation of portal fibroblasts ${ }^{10,18}$ by a poorly understood mechanism. Possible explanations include the increased pressure, cytokine secretion by damaged bile duct cells or by recruited inflammatory cells, or a direct effect of bile acids. Neither cell culture nor animal models allow to easily decipher these mechanisms.

Precision-cut liver slices (PCLS) have been recently used for metabolic and toxicological studies on various liver cell types. ${ }^{19-22}$ The PCLS culture model allows the maintenance of normal lobular architecture and cell-cell interactions within their original matrix. It has recently been applied to the study of hepatic stellate cell activation. ${ }^{23-25}$ This model appears especially well suited to the investigation of the effects of bile acids on biliary epithelial cells and portal fibroblasts since it eliminates two confusing factors, hydrostatic pressure and recruitment of circulating inflammatory cells.

Thus, in this study, we took advantage of the PCLS model to analyze the effects of bile acids on the liver, focusing on biliary epithelial cells and portal fibroblasts.

\section{Materials and methods}

\section{Preparation of Rat PCLS}

PCLS were obtained as previously described. ${ }^{26}$ Male Wistar rats (Charles Rivers, St Aubin les Elbeuf, France) weighing approximately $400 \mathrm{~g}$ were anesthetized with an intraperitoneal injection of ketamine (Imalgen ${ }^{\circledR}$, Rhône Mérieux, Lyon, France) and xylazine (Rompun ${ }^{\circledR}$, Bayer Pharma, Puteaux, France). Liver was perfused in situ $(20 \mathrm{ml} / \mathrm{min})$ with preoxygenated ice-cold Hanks' balanced salt solution (Invitrogen, Cergy Pontoise, France), supplemented with $5 \mathrm{mM}$ glucose and $50 \mu \mathrm{g} / \mathrm{ml}$ gentamycin, via a plastic catheter placed in the portal vein. After $5 \mathrm{~min}$ perfusion, the liver was excised and tissue cores were prepared using a motor-driven coring tool ( $8 \mathrm{~mm}$ diameter). Consecutive PCLS ( $250 \mu \mathrm{m}$ thickness) were obtained using a Krumdieck Tissue Slicer (Alabama Research and Development Corporation, Munford, AL, USA). During preparation, tissue cores and slices were maintained in continuously oxygenated ice-cold Hanks' balanced salt solution.

\section{Rat PCLS Culture and Treatment}

PCLS were placed on a stainless-steel insert (Alabama Research and Development Corporation) in an incubation vial containing culture medium. Vials were set on a roller platform, and gently agitated in a humidified incubator (Sanyo, Osaka, Japan) at $37^{\circ} \mathrm{C}$, with $5 \% \mathrm{CO}_{2}$ and $40 \% \mathrm{O}_{2}$. A central $2 \mathrm{~mm}$ diameter hole in the vial caps allowed gas exchanges. Slices were firstly incubated for $2 \mathrm{~h}$ in Williams' E medium (Invitrogen) supplemented with $0.35 \mu \mathrm{M}$ insulin (Sigma, St-Quentin-Fallavier, France), $0.1 \mu \mathrm{M}$ dexamethasone (Sigma) and 5\% fetal calf serum (FCS; Dutscher, Issy-les-Moulineaux, France) to promote adherence to the mesh and to avoid slice unsticking. After $2 \mathrm{~h}$, PCLS were treated for $48 \mathrm{~h}$ with bile acids. The following sodium salts of tauro-conjugated forms of three major endogenous bile acids with increasing hydrophobicity were tested: taurocholate (TC), taurodeoxycholate (TDC) and taurolithocholate (TLC) (Calbiochem, San Diego, CA, USA) $100 \mu \mathrm{M}$ diluted in ethanol $70 \%$. Incubations were performed in Williams' $\mathrm{E}$ medium supplemented with insulin, dexamethasone and 1\% FCS. In our experiments, the final concentration of ethanol did not exceed $0.3 \%$. In some experiments, bromodeoxyuridine (BrdU; Roche Diagnostics, Meylan, France) was added to the culture medium $1 \mathrm{~h}$ before the end of treatment. In each preparation $(n=12)$, at least eight PCLS per condition were used.

\section{Cytotoxicity Assays}

Overall slice viability was evaluated as follows. After treatment, culture medium was removed and PCLS were incubated in Williams' $E$ medium containing $2 \mathrm{mg} / \mathrm{ml} \quad 3$-(4,5-dimethylthiazol-2-yl)2,5-diphenyltetrazolium bromide (MTT). After $2 \mathrm{~h}$ at $37^{\circ} \mathrm{C}$, PCLS were solubilized with dimethyl sulfoxyde (Merck, Fontenay-sous-Bois, France) and the optical density at $570 \mathrm{~nm}$ was measured in a microplate reader (Labsystems, Vantaa, Finland).

As an additional test, we also measured the leakage of lactate dehydrogenase (LDH) in the culture medium. After treatment, supernatants were collected, centrifuged, and LDH activity released from the cytosol of damaged cells was assessed using the Cytotoxicity Detection Kit LDH (Roche Diagnostics). The optical density at $492 \mathrm{~nm}$ was measured in a microplate reader.

\section{Antibodies}

For immunohistochemistry on formalin-fixed, paraffin-embedded PCLS sections, the following antibodies were used: rabbit polyclonal antibodies against platelet-derived growth factor (PDGF) receptor- $\beta$ diluted 1:100 (Santa Cruz Biotechnology, Santa Cruz, CA, USA), and active caspase 3 diluted 1:600 (R\&D Systems, Minneapolis, MN, USA), a rat monoclonal antibody against BrdU diluted 1:500 (Oxford Biotechnology Immunologicals, Oxford, UK), mouse monoclonal antibodies against proliferating cell nuclear antigen diluted 1:200 (PCNA; DakoCytomation, Trappes, France), and cytokeratin 19 diluted 1:100 (Sigma), and a goat polyclonal antibody against osteopontin diluted 1:500 (Sigma). PDGF receptor- $\beta$ is a well known early marker of 
fibroblastic cell activation. ${ }^{27-29}$ Active caspase 3 has been extensively used to detect cell apoptosis. Cytokeratin $19,{ }^{30}$ and osteopontin ${ }^{31,32}$ are known to be expressed by biliary epithelial cells. For double immunofluorescence on frozen PCLS sections, a monoclonal antibody (IgG1) specific to the ED-A domain of fibronectin diluted 1:100 (Abcam, Cambridge, UK), ${ }^{33}$ and a pan-cytokeratin rabbit polyclonal antibody diluted 1:200 (DakoCytomation) were used.

\section{Histology and Immunostaining}

PCLS were formalin-fixed and paraffin-embedded or immediately frozen in liquid nitrogen-cooled isopentane and stored at $-80^{\circ} \mathrm{C}$. Formalin-fixed, paraffin-embedded PCLS sections ( $5 \mu \mathrm{m}$-thick) were stained with hematoxylin-eosin for routine histology, or processed for immunohistochemistry. Briefly, sections were incubated with primary antibodies, then, after washing, with the anti-rabbit or anti-mouse horseradish peroxidase Envision $^{\mathrm{TM}}+$ system, and finally, with diaminobenzidine (DakoCytomation). Sections were counterstained with hematoxylin or eosin. For PDGF receptor- $\beta$, osteopontin and BrdU stainings, sections were first subjected to steam heat antigen retrieval in citrate buffer $0.01 \mathrm{M}, \mathrm{pH} 6.0$ for $5 \mathrm{~min}$. For BrdU staining, sections were pretreated with $2 \mathrm{M} \mathrm{HCl}$ for $1 \mathrm{~h}$ at $37^{\circ} \mathrm{C}$ and incubated with rabbit anti-rat immunoglobulins diluted 1:100 (DakoCytomation) before anti-rabbit Envision $^{\mathrm{TM}}+$ system detection. For osteopontin, sections were incubated with rabbit anti-goat immunoglobulins diluted 1:100 (DakoCytomation) be-

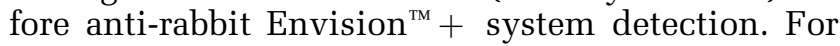
immunofluorescence, frozen PCLS sections $(5 \mu \mathrm{m}$ thick) fixed in cold acetone were incubated with the mouse monoclonal and the rabbit polyclonal antibodies mixed. Oregon green 488-conjugated goat anti-mouse IgG (Molecular Probes, Eugene, OR, USA) and Alexa fluor 594-conjugated goat antirabbit IgG (Molecular Probes) both diluted 1:200 were used for the second step. Nuclei were stained with DAPI (Sigma). Immunostainings were examined with a Zeiss Axioplan 2 microscope (Carl Zeiss Microscopy, Jena, Germany) equipped with epiillumination and specific filters for immunofluorescence analysis. Images were acquired with an AxioCam camera (Carl Zeiss Vision, Hallbergmoos, Germany) by means of the AxioVision image processing and analysis system (Carl Zeiss Vision). Immunostainings with green, red and blue fluorescence were artificially treated using the AxioVision software (Carl Zeiss Vision).

\section{Quantitative Image Analysis}

Parenchyma lesions and portal fibroblast activation (PDGF receptor- $\beta$ staining) were evaluated by quan- titative image analysis. Images were acquired as above and quantitative data were obtained using a computerized image analysis system (KS 300, Carl Zeiss Vision). The analysis was performed on an average of 20 fields (at least four PCLS) per condition using the $\times 10$ objective. Parenchyma lesion and portal fibroblast activation were expressed as a percentage of parenchyma damaged or PDGF receptor- $\beta$-stained areas per field area, respectively. Cellular proliferation was evaluated by counting BrdU-positive nuclei in 10 portal zones (at least four PCLS) per condition with a $\times 20$ objective.

\section{Portal Fibroblast Culture and Treatment}

Rat portal fibroblasts were obtained as previously described. ${ }^{11,12}$ Briefly, the portal tracts were isolated by collagenase digestion of the liver, and then incubated with $0.1 \%$ pronase (Roche Diagnostics). After digestion, portal tract residues were allowed to adhere in Petri dishes and cultured in Dulbeco's minimum essential medium (Invitrogen) in the presence of $10 \%$ FCS at $37^{\circ} \mathrm{C}$ in a humidified atmosphere containing $5 \% \mathrm{CO}_{2}$; after $2-3$ days, an extensive proliferation of fibroblastic cells was observed along biliary structures. Cells between passage 2 and 5 were distributed in 96-wells plates (3000 cells/well). At $24 \mathrm{~h}$ after seeding, cells were treated with TDC, TLC or TC (50 and $100 \mu \mathrm{M}$ ) in the presence of $1 \%$ FCS. After 24,48 or $72 \mathrm{~h}$, the MTT test was used to measure cell proliferation. The presence on portal fibroblasts of the epidermal growth factor receptor (EGFR) was determined by Western blotting with a rabbit polyclonal antibody (Cell Signaling Technology, Beverly, MA, USA) according to Neaud et al. ${ }^{34}$

\section{Statistical Analysis}

Results are expressed as mean values \pm s.e.m. Comparison of multiple means was carried out by one way analysis of variance. A $P<0.05$ was considered significant.

\section{Results}

\section{Effects of Bile Acids on Rat PCLS Viability and Cytolysis (Figure 1a and b)}

After $24 \mathrm{~h}$ of culture, no significant differences in PCLS viability were observed between control and bile acid-treated PCLS as shown with the MTT assay. After $48 \mathrm{~h}$ of culture, PCLS viability decreased when treated with the hydrophobic bile acids, TDC $(P<0.05)$ and TLC $(P<0.001)$, as compared with control slices. No loss in viability was seen with the hydrophilic bile acid TC.

$\mathrm{LDH}$ release assays confirmed these results. Actually, LDH leakage into the supernatant in- 
creased already significantly after $24 \mathrm{~h}$ of culture in TDC- and TLC-treated PCLS as compared with control slices $(P<0.03$ and $P<0.01$, respectively); after TC treatment, LDH leakage remained similar to control PCLS. Similar results were seen after $48 \mathrm{~h}$ of culture with TDC or TLC $(P<0.02$ and $P<0.03$, respectively as compared with control PCLS), whereas TC treatment reduced significantly LDH leakage as compared with control slices $(P<0.04)$.
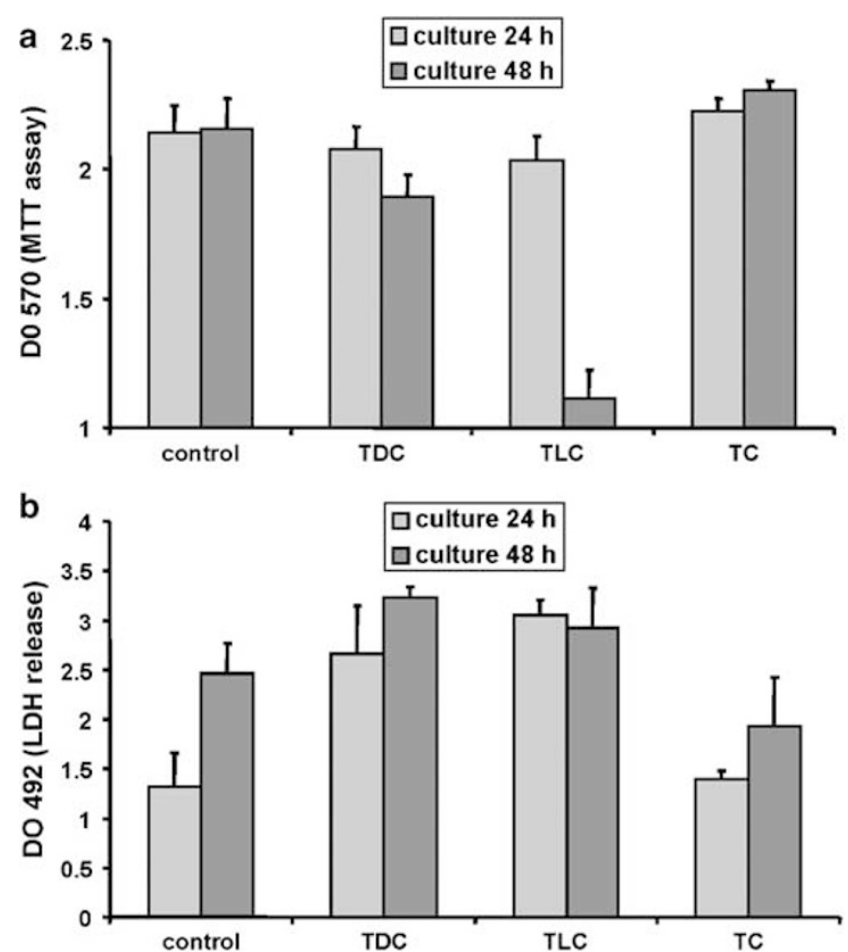

Figure 1 MTT assay (a) and LDH release (b) in bile acid-treated PCLS. MTT assay (a): after $24 \mathrm{~h}$ of culture, no significant differences are observed in bile acid-treated and control PCLS. After $48 \mathrm{~h}$ of culture, as compared with control slices, PCLS viability is decreased after treatment with TDC $(P<0.05)$ and TLC $(P<0.001)$, while after TC treatment, there is a trend towards an increase of PCLS viability (NS, $P<0.07$ ). The parameter directly measured by the test is a mitochondrial enzyme activity, which is related with the viability. LDH release (b): after $24 \mathrm{~h}$ of culture, as compared with control slices, LDH leakage into the supernatant increases in TDC- and TLC-treated PCLS $(P<0.03$ and $P<0.01$, respectively) and is not modified after TC treatment. After $48 \mathrm{~h}$ of culture, LDH leakage increases after treatment with TDC $(P<0.02)$ or TLC $(P<0.03)$, while when treated with TC, LDH leakage decreases significantly $(P<0.04)$ as compared with control. The graphs show the mean values obtained from five different preparations of PCLS. In each preparation, at least four PCLS per point were used.

\section{Effects of Bile Acids on Hepatocyte Survival in Rat PCLS}

Histological examination of control PCLS after $48 \mathrm{~h}$ of culture showed a well-preserved parenchyma with no damaged zones (Figure 2a) and absence of active caspase 3 expression (Figure 2b). TDC-treated PCLS showed focal damaged areas in the whole parenchyma (Figure 2c) with apoptotic hepatocytes, as shown by a positive labelling with the active caspase 3 antibody (Figure 2d). TLC strongly induced hepatocyte necrosis and apoptosis with a mosaic distribution (Figure 2e and f). With both TDC and TLC, areas with damaged hepatocytes affected $20 \pm 5 \%$ of the parenchyma after $24 \mathrm{~h}$ of culture, and $50 \pm 8 \%$ of the parenchyma after $48 \mathrm{~h}$ of culture. On the contrary, PCLS treated with TC appeared very well preserved with no necrotic or apoptotic cells (Figure 2g and h). In TLC-treated PCLS, characteristic features of apoptosis were observed with chromatin condensation (Figure 3a); active caspase 3 was detected with a typical pattern in these apoptotic hepatocytes (Figure 3b). Similar features were observed in apoptotic hepatocytes after TDC treatment (data not shown).

\section{Effects of Bile Acids on Biliary Epithelial Cell Survival and Proliferation, and on Portal Fibroblast Activation in Rat PCLS}

As early as $24 \mathrm{~h}$ of exposure, portal tracts were enlarged with an increase in the number of biliary ductular structures in TDC- and TC-treated PCLS compared with nontreated and TLC-treated PCLS; after $48 \mathrm{~h}$ of culture, this portal tract enlargement was obvious (Figure 2c and g). In nontreated and TLC-treated PCLS, no BrdU staining was detected after $24 \mathrm{~h}$ of culture; very few BrdU-positive nuclei were detected after $48 \mathrm{~h}$ of culture (Figure $4 \mathrm{a}$ ). In TDC- and TC-treated PCLS, after $24 \mathrm{~h}$ of culture, very few BrdU-positive nuclei were detected within portal areas; after $48 \mathrm{~h}$ of culture, BrdU staining showed numerous proliferating cells $(20 \pm 3$ cells per portal zone), including fibroblastic cells (about $70 \%$ of BrdU-positive cells) and biliary epithelial cells, within portal areas (Figure 4b). Immunostaining with PCNA confirmed cell proliferation in portal tracts of TDC- and TC-treated PCLS (data not shown). In addition, TDC and TC treatment led to portal fibroblast activation as judged from staining for PDGF receptor- $\beta$. Indeed, after $48 \mathrm{~h}$ of culture, in nontreated (Figure 4c) and TLC-treated (Figure 4d) PCLS, PDGF receptor- $\beta$ expression was limited to

Figure 2 Hematoxylin-eosin staining (a, c, e, g) and active caspase 3 expression (b, d, f, h) in bile acid-treated PCLS. After 48 h of culture, in control PCLS, parenchyma is well preserved with no necrotic zones detected (a) and absence of active caspase 3 expression (b). TDCtreated PCLS show focal necrosis areas in the whole parenchyma (c) and apoptotic hepatocytes expressing active caspase 3 (d). TLC strongly induces hepatocyte necrosis and apoptosis with a mosaic distribution (e and f). PCLS treated with TC appear to be very well preserved with no necrotic or apoptotic cells detected ( $\mathbf{g}$ and $\mathbf{h}$ ). Portal tracts are enlarged with an increase of the number of biliary ductular structures in TDC- and TC-treated PCLS (c and g, arrows). 

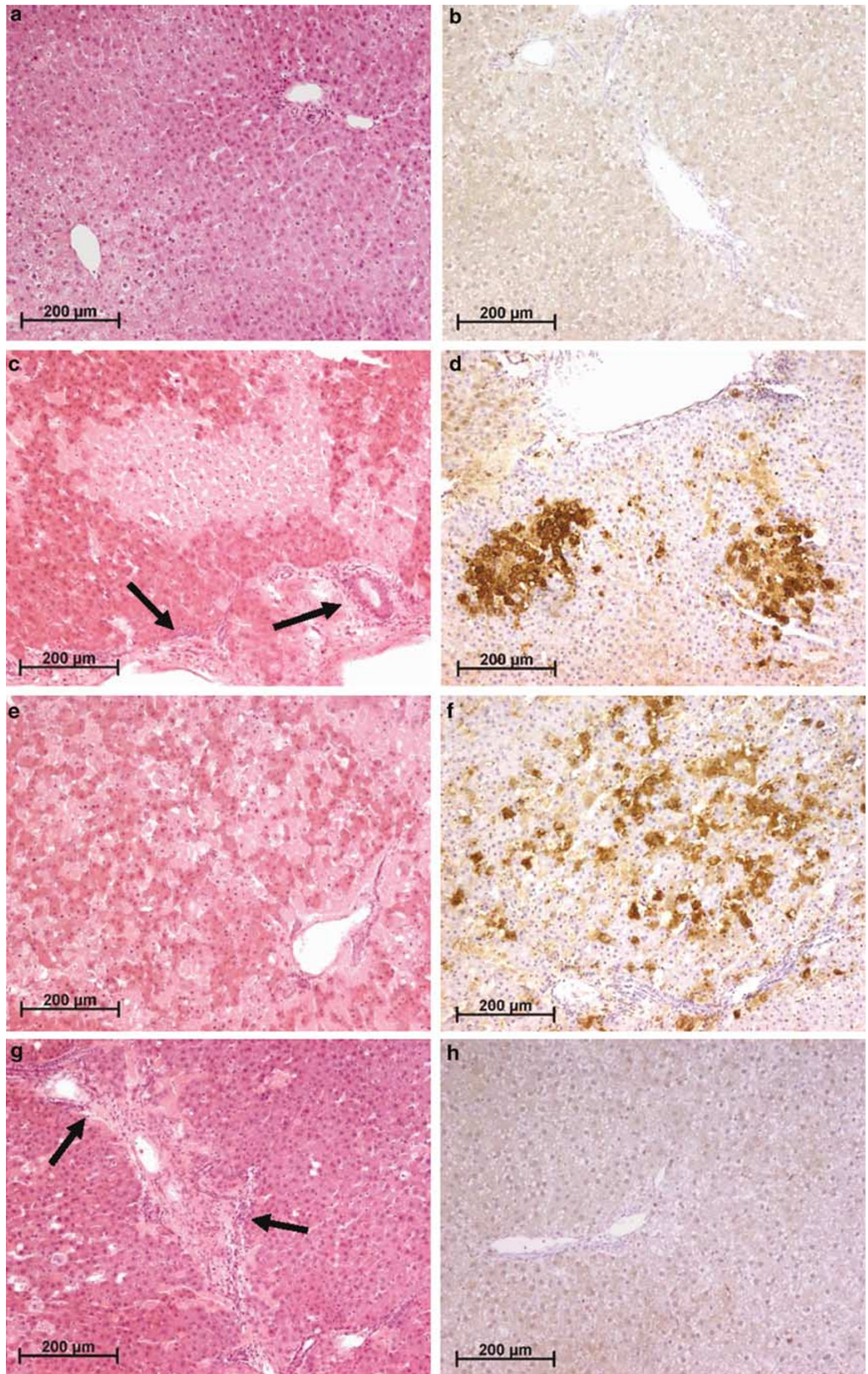
centrolobular veins and to a few mesenchymal cells of the portal tract stroma and vessels $(1.1 \pm 0.2 \%$ per field area); in contrast, a strong PDGF receptor- $\beta$ expression was observed in portal fibroblastic cells around proliferating ductular structures of TDC- and TC-treated slices $(5.6 \pm 0.5$ and $11.3 \pm 1.2 \%$, respectively; $P<0.02$ and $P<0.001$, respectively, compared with control) (Figure $4 \mathrm{e}$ and f). Portal fibroblast activation was confirmed using ED-A fibronectin expression. After $48 \mathrm{~h}$ of culture, in
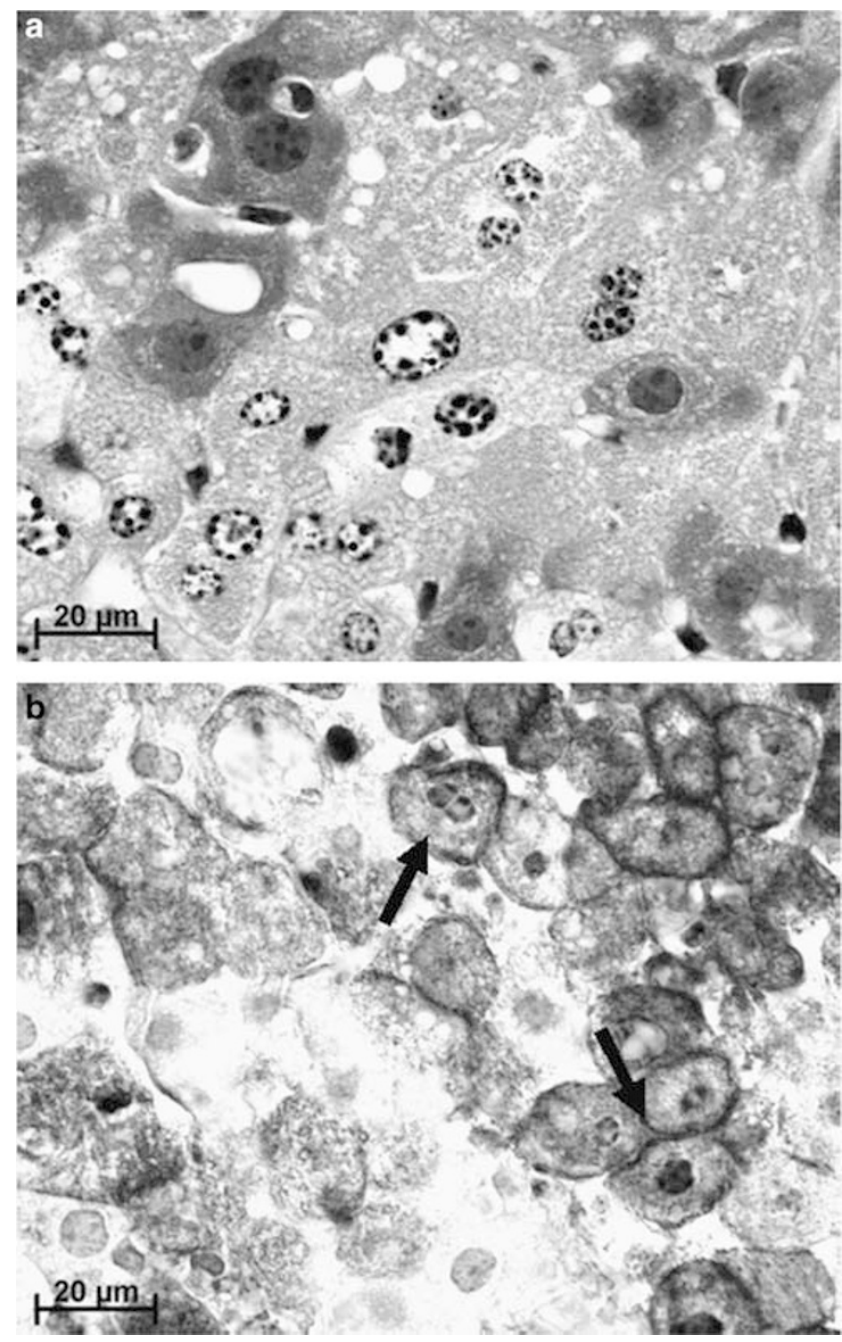

Figure 3 Apoptotic features in hepatocytes of TLC-treated rat PCLS. In (a) hematoxylin-eosin staining underlines the characteristic organization of the chromatin in apoptotic hepatocytes. In (b) active caspase 3-expressing hepatocytes show typical features of apoptotic cells (arrows). portal tract of nontreated slices, ED-A fibronectin was expressed exclusively in vessels (Figure 4g); in TC-treated slices, ED-A fibronectin was observed in portal fibroblasts involved in portal tract enlargement (Figure 4h). After $24 \mathrm{~h}$ of culture with TLC, numerous apoptotic bodies were observed into the portal tracts, corroborated by active caspase 3 immunostaining; apoptotic cells were clearly observed in ductular structures (Figure 5a). In fact, after $48 \mathrm{~h}$ of culture, no more biliary structures were observed, as underlined by the absence of osteopontin expression in portal tracts (Figure 5b) in contrast with TC-treated PCLS where numerous biliary structures expressing osteopontin were clearly observed (Figure 5c). Immunostaining with cytokeratin 19 confirmed the disappearance of biliary epithelial cells in TLC-treated PCLS (data not shown).

\section{Effects of Bile Acids in Culture of Isolated Rat Portal Fibroblasts}

In order to elucidate if bile acids have a direct effect on portal fibroblasts, cultured portal fibroblasts were treated with TDC, TLC and TC (50 and $100 \mu \mathrm{M}$ ) for 24,48 or $72 \mathrm{~h}$. In our culture conditions (1\% FCS), the number of cells was not significantly modified in nontreated cells after 24, 48 and $72 \mathrm{~h}$ of culture (data not shown). In portal fibroblasts treated with bile acids during 24, 48 (data not shown), or $72 \mathrm{~h}$ (Figure 6), proliferation was not significantly induced compared with nontreated cells. Cultured portal fibroblasts expressed the EGFR (Figure 6, inset).

\section{Discussion}

In this study, we show that TDC and TLC, two hydrophobic bile acids, induced hepatocyte necrosis and apoptosis, whereas TC, an hydrophilic bile acid, improved slice viability as compared with controls. Both TDC and TC induced biliary epithelial cell proliferation, together with proliferation and activation of portal fibroblasts, as shown by PDGF receptor- $\beta^{27-29}$ and ED-A fibronectin ${ }^{11,18}$ expression. TLC induced biliary epithelial cell apoptosis with little portal fibroblast activation.

The exact mechanisms involved in bile acidinduced cell death, in hepatocytes and biliary epithelial cells, remain unexplained. It has been shown recently that oncosis represents the main

\footnotetext{
Figure 4 Portal cell proliferation, PDGF receptor- $\beta$ and ED-A fibronectin expression in bile acid-treated PCLS. After $48 \mathrm{~h}$ of culture, compared with nontreated PCLS (a), BrdU staining shows in TC-treated PCLS (b), an increase of proliferating cells within portal areas, including fibroblastic cells and biliary epithelial cells (b). A slight PDGF receptor- $\beta$ expression is detected in portal tract of nontreated (c) and TLC-treated (d) PCLS; in TDC- and TC-treated slices (e and $\mathbf{f}$, respectively), a strong expression of PDGF receptor- $\beta$ is observed in numerous portal fibroblastic cells around proliferating ductular structures. Using double immunofluorescence, after $48 \mathrm{~h}$ of culture, in nontreated PCLS (g), cytokeratin (in red) is detected in hepatocytes and biliary epithelial cells (arrow), and ED-A fibronectin (in green) is present exclusively in the wall of a portal vessel; in TC-treated PCLS (h), ED-A fibronectin expression is increased in enlarged portal tracts and in portal fibroblasts which expand into the parenchyma.
} 

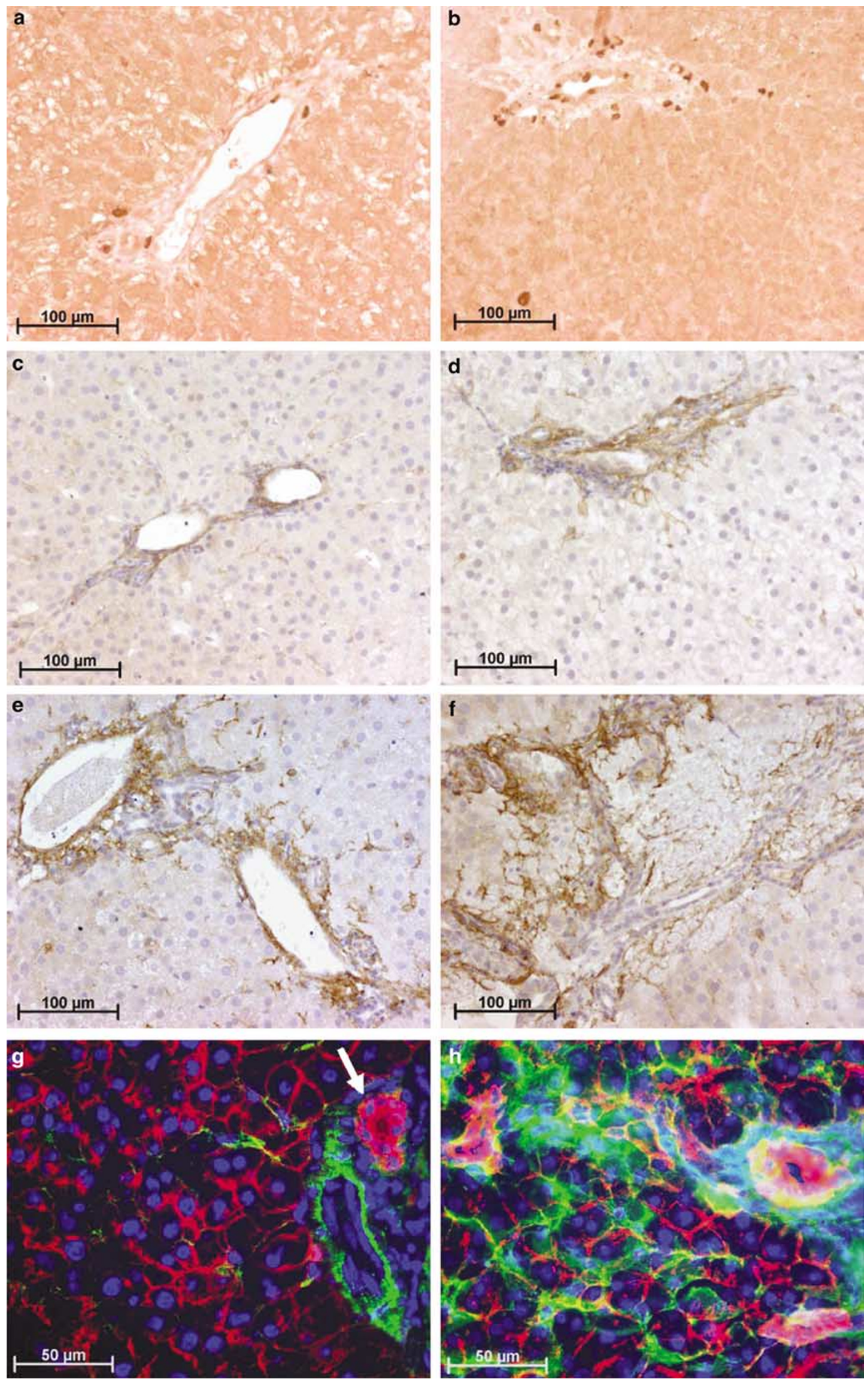
282
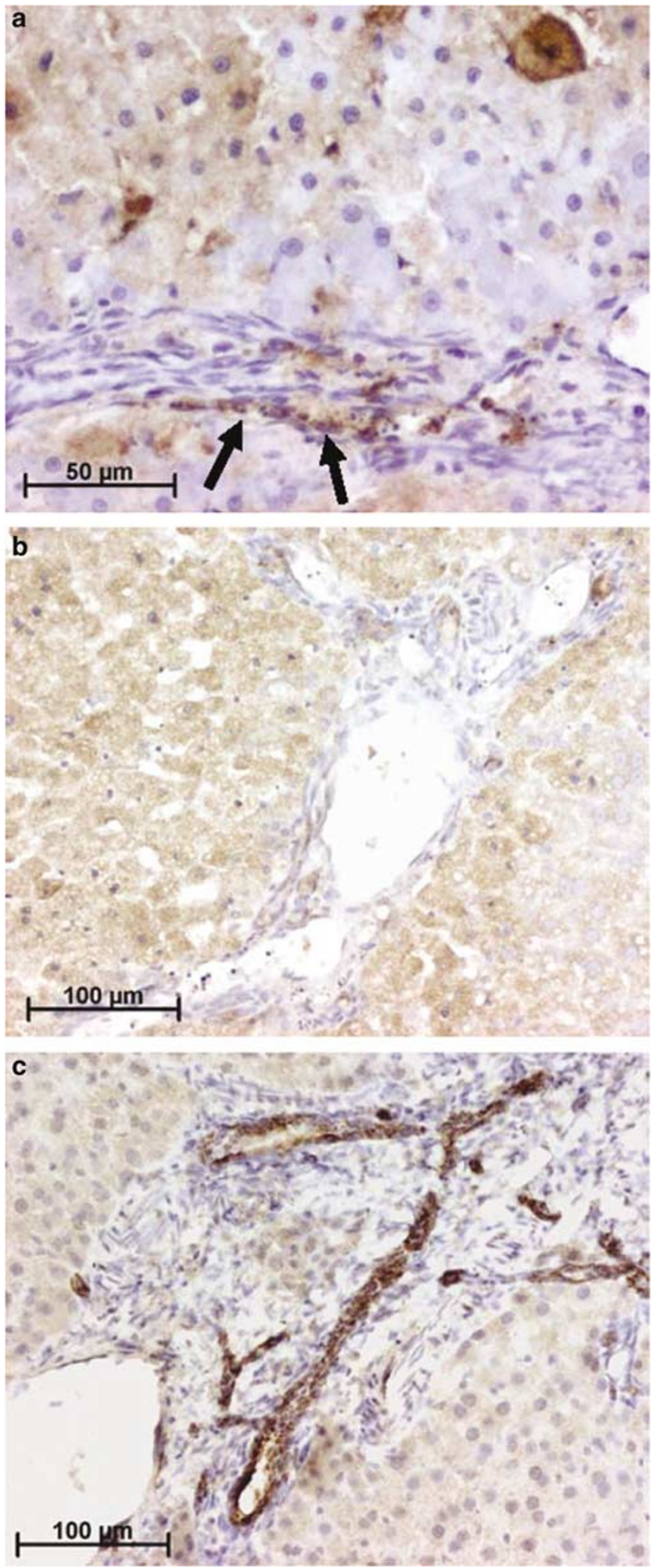

Figure 5 Biliary epithelial cell apoptosis in TLC-treated PCLS, and osteopontin expression in TLC- and TC-treated PCLS. After $24 \mathrm{~h}$ of culture with TLC, an active caspase 3 expression is present into the portal tracts in ductular structures (a, arrows); after $48 \mathrm{~h}$ of culture with TLC, the absence of osteopontin expression in portal tracts underlines the disappearance of biliary structures (b). In contrast, in TC-treated PCLS, osteopontin expression allows the detection of the numerous biliary structures (c).

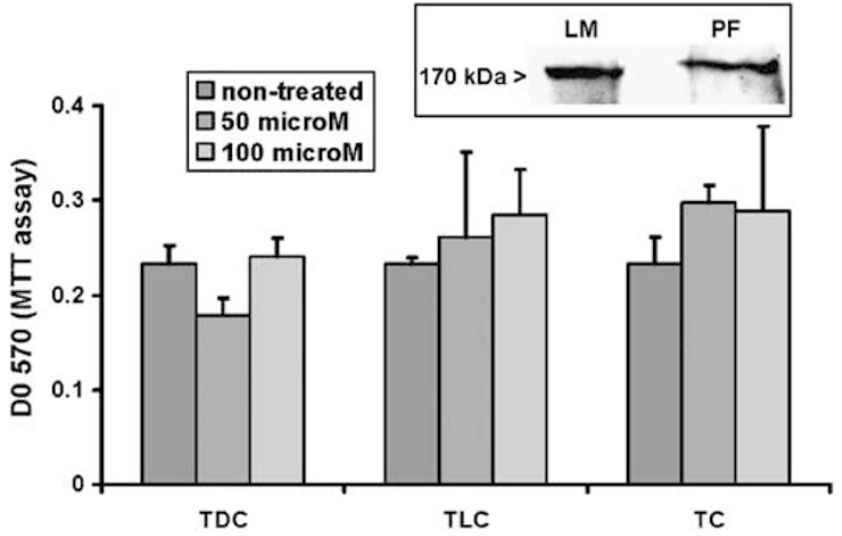

Figure 6 Proliferation of cultured rat portal fibroblasts treated with bile acids. No significant proliferation was induced in cultured portal fibroblasts treated with bile acids during $72 \mathrm{~h}$ compared with nontreated cells. The graph shows the mean values obtained from three experiments. In each experiment, at least three measurements per point were performed. Cultured portal fibroblasts (PF) express the EGFR (inset); as a positive control, human liver myofibroblasts (LM) are shown (inset).

type of hepatocyte death in acute cholestasis in mice. ${ }^{35}$ We suggest that the relevant PCLS model described here could help in the critical analysis of the precise mechanisms leading to cell death in cholestatic liver diseases. Anyhow, these results obtained in the PCLS model confirm our previous observations showing that, in cultured biliary epithelial cells, TDC markedly induced biliary epithelial cell proliferation while TLC induced biliary epithelial cell apoptosis. ${ }^{36}$ It has been shown that hydrophobic bile acids are able to solubilize polar lipids, such as phospholipids and cholesterol, leading to the disruption of plasma membranes; ${ }^{37}$ this explains at least in part the correlation between bile acid hydrophobicity and cytotoxicity. In addition, we now show that biliary epithelial cell proliferation induced by TDC and TC is accompanied by portal fibroblast proliferation and activation. Moreover, we observe an early expression of ED-A fibronectin which is necessary for the induction of the myofibroblastic phenotype by transforming growth factor- $\beta 1 ;^{38}$ we had shown that ED-A fibronectin is expressed early during the activation of portal fibroblasts in vitro, ${ }^{11}$ and after bile duct ligation. ${ }^{18}$ These data were obtained with rat PCLS; however, preliminary observations with mouse PCLS suggest that bile acids show comparable effects on rat and mouse PCLS (data not shown).

After bile duct ligation, proliferation of biliary structures is associated with a proliferation of portal fibroblasts forming concentric layers around bile ductules; these portal fibroblasts acquire a myofibroblastic phenotype, ${ }^{10-12}$ and are involved in the early deposition of extracellular matrix in the portal zones. ${ }^{18}$ Portal fibroblast activation in this setting may be related to the increased pressure within 
biliary structures as suggested by Slott et $a l,{ }^{16}$ to direct effects of bile acids or to signals originating from biliary epithelial cells or infiltrating inflammatory cells, although the latter are scarce in this model. In our model of cultured PCLS, processes depending on inflammation or biliary pressure cannot be involved; we thus propose that bile acid treatment induces biliary epithelial cell stimulation leading to portal fibroblast proliferation and activation. This is supported by previous results indicating that biliary epithelial cells synthesize a series of relevant cytokines such as monocyte chemotactic protein-1 (MCP-1), ${ }^{39}$ PDGF, ${ }^{12,40}$ transforming growth factor- $\beta,{ }^{41,42}$ and connective tissue growth factor. ${ }^{43}$ The expression of all these cytokines is increased in either experimental or human cholestatic diseases (for review, see ${ }^{44}$ ). Besides its major role in the recruitment and activation of monocytes and $\mathrm{T}$ lymphocytes, ${ }^{45,46}$ MCP-1 is also chemotactic for hepatic stellate cells. ${ }^{47}$ MCP-1 expression, confined to few hepatic stellate cells and biliary epithelial cells in normal liver, is markedly increased within portal tracts during active hepatic fibrogenesis, especially in biliary epithelial cells. ${ }^{39}$ We have shown that biliary epithelial cells treated with TC secrete high amounts of MCP- $1,{ }^{36}$ which makes it a good candidate for portal fibroblast activation in our model. Among other cytokines secreted by biliary epithelial cells, PDGF, ${ }^{48}$ transforming growth factor$\beta,{ }^{49}$ and connective tissue growth factor, ${ }^{50}$ play major roles during hepatic fibrogenic cell activation, by their effects on proliferation, migration and/or extracellular matrix synthesis.

Furthermore, during cholestasis, portal fibroblasts are also exposed to bile acids retained in high amount. Our data with PCLS do not allow to completely exclude a direct effect of bile acids on portal fibroblasts. Recently, it has been shown that hepatic stellate cells express the nuclear factor farnesoid X receptor which is activated by bile acids ${ }^{51}$ portal fibroblasts also express the farnesoid $\mathrm{X}$ receptor (unpublished data). However, whereas bile acids were shown to stimulate the growth of hepatic stellate cells via activation of the EGFR, ${ }^{52}$ we found that they did not induce proliferation of cultured portal fibroblasts which however express the EGFR; this suggests that the hepatic stellate cells used in that work and the portal fibroblasts studied here must probably belong to different cell populations. ${ }^{53}$

In conclusion, our results indicate that individual bile acids induce cell type-specific effects in a complex liver microenvironment. These data appear particularly relevant in human pathological situations such as cholestatic liver diseases, in which bile duct damage and portal fibrogenesis are hallmarks of these diseases. ${ }^{54}$ The fact that PCLS support biliary epithelial cell and portal fibroblast proliferation will make this model very useful for the study of the mechanisms involved in portal fibrosis.

\section{Acknowledgements}

This work was supported in part by Région Aquitaine. Christelle Guyot was recipient of a fellowship from Région Aquitaine.

\section{References}

1 Schmucker DL, Ohta M, Kanai S, et al. Hepatic injury induced by bile salts: correlation between biochemical and morphological events. Hepatology 1990;12: 1216-1221.

2 Poupon R, Chazouilleres O, Poupon RE. Chronic cholestatic diseases. J Hepatol 2000;32(Suppl 1): 129-140.

3 Greim H, Czygan P, Schaffner F, et al. Determination of bile acids in needle biopsies of human liver. Biochem Med 1973;8:280-286.

4 Scholmerich J, Becher MS, Schmidt K, et al. Influence of hydroxylation and conjugation of bile salts on their membrane-damaging properties-studies on isolated hepatocytes and lipid membrane vesicles. Hepatology 1984;4:661-666.

5 Sokol RJ, Devereaux M, Khandwala R, et al. Evidence for involvement of oxygen free radicals in bile acid toxicity to isolated rat hepatocytes. Hepatology 1993; 17:869-881

6 Heuman DM, Mills AS, McCall J, et al. Conjugates of ursodeoxycholate protect against cholestasis and hepatocellular necrosis caused by more hydrophobic bile salts. In vivo studies in the rat. Gastroenterology 1991;100:203-211.

7 Delzenne NM, Calderon PB, Taper HS, et al. Comparative hepatotoxicity of cholic acid, deoxycholic acid and lithocholic acid in the rat: in vivo and in vitro studies. Toxicol Lett 1992;61:291-304.

8 Faubion WA, Guicciardi ME, Miyoshi H, et al. Toxic bile salts induce rodent hepatocyte apoptosis via direct activation of Fas. J Clin Invest 1999;103:137-145.

9 Lotersztajn S, Julien B, Teixeira-Clerc F, et al. Hepatic fibrosis: molecular mechanisms and drug targets. Annu Rev Pharmacol Toxicol 2005;45:605-628.

10 Tuchweber B, Desmoulière A, Bochaton-Piallat ML, et al. Proliferation and phenotypic modulation of portal fibroblasts in the early stages of cholestatic fibrosis in the rat. Lab Invest 1996;74:265-278.

11 Uchio K, Tuchweber B, Manabe N, et al. Cellular retinol-binding protein-1 expression and modulation during in vivo and in vitro myofibroblastic differentiation of rat hepatic stellate cells and portal fibroblasts. Lab Invest 2002;82:619-628.

12 Kinnman N, Francoz C, Barbu V, et al. The myofibroblastic conversion of peribiliary fibrogenic cells distinct from hepatic stellate cells is stimulated by platelet-derived growth factor during liver fibrogenesis. Lab Invest 2003;83:163-173.

13 Roberts SK, Ludwig J, Larusso NF. The pathobiology of biliary epithelia. Gastroenterology 1997;112:269-279.

14 Alpini G, Lenzi R, Sarkozi L, et al. Biliary physiology in rats with bile ductular cell hyperplasia. Evidence for a secretory function of proliferated bile ductules. J Clin Invest 1988;81:569-578.

15 Marucci L, Baroni GS, Mancini R, et al. Cell proliferation following extrahepatic biliary obstruction. Evaluation 
by immunohistochemical methods. J Hepatol 1993;17: 163-169.

16 Slott PA, Liu MH, Tavoloni N. Origin, pattern, and mechanism of bile duct proliferation following biliary obstruction in the rat. Gastroenterology 1990;99:466-477.

17 Benedetti A, Alvaro D, Bassotti C, et al. Cytotoxicity of bile salts against biliary epithelium: a study in isolated bile ductule fragments and isolated perfused rat liver. Hepatology 1997;26:9-21.

18 Desmoulière A, Darby I, Monte Alto Costa A, et al. Extracellular matrix deposition, lysyl oxydase expression, and myofibroblastic differentiation during the initial stages of cholestatic fibrosis in the rat. Lab Invest 1997;76:765-778.

19 Lerche-Langrand C, Toutain HJ. Precision-cut liver slices: characteristics and use for in vitro pharmacotoxicology. Toxicology 2000;153:221-253.

20 Olinga P, Hof IH, Merema MT, et al. The applicability of rat and human liver slices to the study of mechanisms of hepatic drug uptake. J Pharmacol Toxicol Methods 2001;45:55-63.

21 Olinga P, Merema MT, de Jager MH, et al. Rat liver slices as a tool to study LPS-induced inflammatory response in the liver. J Hepatol 2001;35:187-194.

22 Neyrinck AM, Margagliotti S, Delzenne NM. Insight into the involvement of Kupffer cell-derived mediators in the hepatoprotective effect of glycine upon inflammation: study on rat precision-cut liver slices. Inflamm Res 2005;54:106-112.

23 Verrill C, Davies J, Millward-Sadler H, et al. Organotypic liver culture in a fluid-air interface using slices of neonatal rat and adult human tissue-a model of fibrosis in vitro. J Pharmacol Toxicol Methods 2002;48: 103-110.

24 Vickers AE, Saulnier M, Cruz E, et al. Organ slice viability extended for pathway characterization: an in vitro model to investigate fibrosis. Toxicol Sci 2004; 82:534-544.

25 Van de Bovenkamp M, Groothuis GM, Draaisma AL, et al. Precision-cut liver slices as a new model to study toxicity-induced hepatic stellate cell activation in a physiologic milieu. Toxicol Sci 2005;85:632-638.

26 Moronvalle-Halley V, Sacre-Salem B, Sallez V, et al. Evaluation of cultured, precision-cut rat liver slices as a model to study drug-induced liver apoptosis. Toxicology 2005;207:203-214.

27 Wong L, Yamasaki G, Johnson RJ, et al. Induction of beta-platelet-derived growth factor receptor in rat hepatic lipocytes during cellular activation in vivo and in culture. J Clin Invest 1994;94:1563-1569.

28 Pinzani M, Milani S, Grappone C, et al. Expression of platelet-derived growth factor in a model of acute liver injury. Hepatology 1994;19:701-707.

29 Pinzani M, Milani S, Herbst H, et al. Expression of platelet-derived growth factor and its receptors in normal human liver and during active hepatic fibrogenesis. Am J Pathol 1996;148:785-800.

30 LeSage G, Glaser S, Alpini G. Regulation of cholangiocyte proliferation. Liver 2001;21:73-80.

31 Brown LF, Berse B, Van de Water L, et al. Expression and distribution of osteopontin in human tissues: widespread association with luminal epithelial surfaces. Mol Biol Cell 1992;3:1169-1180.

32 Lorena D, Darby IA, Gadeau AP, et al. Osteopontin expression in normal and fibrotic liver. Altered liver healing in osteopontin-deficient mice. J Hepatol 2006, in press.
33 Borsi L, Carnemolla B, Castellani P, et al. Monoclonal antibodies in the analysis of fibronectin isoforms generated by alternative splicing of mRNA precursors in normal and transformed human cells. J Cell Biol 1987;104:595-600.

34 Neaud V, Duplantier JG, Mazzocco C, et al. Thrombin up-regulates tissue factor pathway inhibitor-2 synthesis through a cyclooxygenase-2-dependent, epidermal growth factor receptor-independent mechanism. J Biol Chem 2004;279:5200-5206.

35 Fickert P, Trauner M, Fuchsbichler A, et al. Oncosis represents the main type of cell death in mouse models of cholestasis. J Hepatol 2005;42:378-385.

36 Lamireau T, Zoltowska M, Levy E, et al. Effects of bile acids on biliary epithelial cells: proliferation, cytotoxicity, and cytokine secretion. Life Sci 2003;72: 1401-1411.

37 Heuman DM, Bajaj RS, Lin Q. Adsorption of mixtures of bile salt taurine conjugates to lecithin-cholesterol membranes: implications for bile salt toxicity and cytoprotection. J Lipid Res 1996;37:562-573.

38 Serini G, Bochaton-Piallat ML, Ropraz P, et al. The fibronectin domain ED-A is crucial for myofibroblastic phenotype induction by transforming growth factorbeta1. J Cell Biol 1998;142:873-881.

39 Marra F, DeFranco R, Grappone C, et al. Increased expression of monocyte chemotactic protein-1 during active hepatic fibrogenesis: correlation with monocyte infiltration. Am J Pathol 1998;152:423-430.

40 Grappone C, Pinzani M, Parola M, et al. Expression of platelet-derived growth factor in newly formed cholangiocytes during experimental biliary fibrosis in rats. J Hepatol 1999;31:100-109.

41 Milani S, Herbst H, Schuppan D, et al. Transforming growth factors beta 1 and beta 2 are differentially expressed in fibrotic liver disease. Am J Pathol 1991; 139:1221-1229.

42 Lamireau T, Le Bail B, Boussarie L, et al. Expression of collagens type I and IV, osteonectin and transforming growth factor beta-1 (TGFbeta1) in biliary atresia and paucity of intrahepatic bile ducts during infancy. J Hepatol 1999;31:248-255.

43 Sedlaczek N, Jia JD, Bauer M, et al. Proliferating bile duct epithelial cells are a major source of connective tissue growth factor in rat biliary fibrosis. Am J Pathol 2001;158:1239-1244.

44 Bataller R, Brenner DA. Liver fibrosis. J Clin Invest 2005;115:209-218.

45 Valente AJ, Graves DT, Vialle-Valentin CE, et al. Purification of a monocyte chemotactic factor secreted by nonhuman primate vascular cells in culture. Biochemistry 1988;27:4162-4168.

46 Carr MW, Roth SJ, Luther E, et al. Monocyte chemoattractant protein 1 acts as a T-lymphocyte chemoattractant. Proc Natl Acad Sci USA 1994;91:3652-3656.

47 Marra F, Romanelli RG, Giannini C, et al. Monocyte chemotactic protein-1 as a chemoattractant for human hepatic stellate cells. Hepatology 1999;29: 140-148.

48 Pinzani M, Gesualdo L, Sabbah GM, et al. Effects of platelet-derived growth factor and other polypeptide mitogens on DNA synthesis and growth of cultured rat liver fat-storing cells. J Clin Invest 1989; 84:1786-1793.

49 Win KM, Charlotte F, Mallat A, et al. Mitogenic effect of transforming growth factor-beta 1 on human Ito cells in culture: evidence for mediation by endogenous 
platelet-derived growth factor. Hepatology 1993;18: 137-145.

50 Paradis V, Dargere D, Bonvoust F, et al. Effects and regulation of connective tissue growth factor on hepatic stellate cells. Lab Invest 2002;82:767-774.

51 Fiorucci S, Antonelli E, Rizzo G, et al. The nuclear receptor SHP mediates inhibition of hepatic stellate cells by FXR and protects against liver fibrosis. Gastroenterology 2004;127:1497-1512.
52 Svegliati-Baroni G, Ridolfi F, Hannivoort R, et al. Bile acids induce hepatic stellate cell proliferation via activation of the epidermal growth factor receptor. Gastroenterology 2005;128:1042-1055.

53 Guyot C, Lepreux S, Combe C, et al. Hepatic fibrosis and cirrhosis: the (myo)fibroblastic cell subpopulations involved. Int J Biochem Cell Biol 2006;38:135-151.

$54 \mathrm{Li} \mathrm{MK}$, Crawford JM. The pathology of cholestasis. Semin Liver Dis 2004;24:21-42. 